\title{
Faecal calprotectin - the Crohn's disease activity predictor of the future
}

\author{
Author: Priyanka Ramphul
}

\section{Introduction}

Crohn's disease (CD) affects at least 115,000 people living in the UK. There are a range of treatments available to control the disease and improve the quality of life of patients. Predicting and monitoring the response of CD to treatment remains difficult.

\section{Case report}

$\mathrm{Mr} \mathrm{X}$ is a 24-year old Caucasian male diagnosed with CD 2 years ago. Despite active medical treatment, he suffers from frequent acute exacerbations. Predicting CD activity before the onset of clinical symptoms would be very useful so Mr X's treatment can be optimised. Routine inflammatory markers (ESR/CRP) are non-specific to CD. Currently, colonoscopy and CT imaging have been used to assess the extent of his mucosal inflammation. This is expensive and resource intensive.

\section{Discussion}

We propose using faecal calprotectin (FC) as a CD activity predictor. FC is a cytosolic protein released by activated neutrophils, in the small and large intestine. It is a surrogate marker of intestinal inflammation. FC is currently being used to screen for $\mathrm{CD}$, but has the potential to become a means of assessing disease evolution with treatment.

If a cut-off of $250 \mu \mathrm{g} / \mathrm{g}$ is applied, $\mathrm{FC}$ has a $90 \%$ sensitivity and $76 \%$ specificity of determining quiescence of CD. ${ }^{1} \mathrm{~A}$ value above $250 \mu \mathrm{g} / \mathrm{g}$ is hence suggestive of active mucosal disease activity. It can be used to monitor disease activity and remission. An increase in FC levels precedes clinical symptoms.

Raised FC correlates with colonoscopy and biopsy findings. As such, it can be a cost-effective investigation to reduce the number of colonoscopies performed.

Using FC has a few limitations. FC is relatively less sensitive at predicting relapse in small bowel $\mathrm{CD}$, compared to Crohn's colitis. There is the risk of false-positive results with FC, this being linked to the use of non-steroidal anti-inflammatory drugs, concurrent infective diarrhoea, diverticular disease and gastrointestinal carcinoma.
But FC still holds enormous potential. For patients with FC levels greater than $250 \mu \mathrm{g} / \mathrm{g}$ and appearing to be in clinical remission, $85 \%$ are likely to have a relapse in the following year, compared to $12 \%$ in patients who have FC levels below $250 \mu \mathrm{g} / \mathrm{g}^{2}$

\section{Conclusion}

Serial measurements of FC allow the identification of patients in whom persistent high levels indicate increased risks of bowel wall damage, and of abscess and stricture formation. FC is a quick test which is cheap, non-invasive and can be easily measured at frequent intervals. It has the potential to become the $\mathrm{CD}$ activity predictor in the near future.

\section{References}

1 Dhaliwal A, Zeino Z, Tomkins C et al. Utility of faecal calprotectin in inflammatory bowel disease (IBD): what cut-offs should we apply? Frontline Gastroenterol 2015;6:14-19.

2 Tibble JA, Sigthorsson G, Bridger S, Fagerhol MK, Bjarnason I. Surrogate markers of intestinal inflammation are predictive of relapse in patients with inflammatory bowel disease. Gastroenterology 2000;119:15-22. 